ARTÍCULOS

\title{
Resiliencia, aprendizaje virtual y COVID-19 en estudiantes universitarios
}

\author{
Resilience and virtual learning against COVID-19 in \\ university students
}

\section{Sanabria Rojas, Liz Gabriela; Farfán Pimentel, Johnny Félix}

\author{
D Liz Gabriela Sanabria Rojas \\ Universidad César Vallejo, Perú \\ lizsanabriarojas@gmail.com \\ (iD) Johnny Félix Farfán Pimentel \\ jfarfanp@ucv.edu.pe \\ Universidad César Vallejo, Perú
}

\section{Delectus}

Instituto Nacional de Investigación y Capacitación Continua, Perú ISSN-e: 2663-1148

Periodicidad: Semestral

núm. 2, 2021

publicaciones.iniccperu@gmail.com

Recepción: 13 Marzo 2021

Aprobación: 01 Junio 2021

Publicación: 01 Julio 2021

Esta licencia permite a otros entremezclar, ajustar y construir a partir de su obra con fines no comerciales, y aunque en sus nuevas creaciones deban reconocerle su autoría y no puedan ser utilizadas de manera comercial, no tienen que estar bajo una licencia con los mismos términos.

\section{cc) (i) (8)}

Esta obra está bajo una Licencia Creative Commons Atribución- NoComercial 4.0 Internacional.
Resumen: El presente artículo muestra un análisis de la bibliografía existente sobre la resiliencia, aprendizaje virtual y COVID-19 con la finalidad de estructurar y comparar los principales temas que fueron cubiertos en su momento, de esta manera poder plantear nuevas ideas de investigación, para ofrecer información acertada al mundo investigativo. La resiliencia es considerada como un aspecto esencial del bienestar que juega un papel importante en la educación, conociendo que varios factores personales y sociales afectan la resiliencia, además del aprendizaje virtual que pasan hacer herramientas de enseñanza en beneficio de la educación. Es necesario prestar atención al desarrollo curricular y utilizar nuevos métodos educativos como la educación virtual para fortalecer la competencia en alfabetización informacional en estudiantes universitarios y de cómo sería la educación ante la crisis originada por la COVID-19. Es por ello que este estudio investigó el efecto de la resiliencia en estudiantes universitarios para un aprendizaje virtual en tiempos de pandemia. Para finalizar propone algunas alternativas de solución para investigaciones futuras como la práctica de la resiliencia y su relación el aprendizaje virtual en tiempos de pandemia. Como consecuencia de esta crisis, cobra mucha importancia la actitud que pone todo estudiante para poder aprovechar y lograr un aprendizaje en línea; de esta manera se sugiere que se debe aprovechar la buena actitud del estudiante para poder salir adelante ante cualquier eventualidad.

Palabras clave: Resiliencia, aprendizaje virtual, COVID-19.

Abstract: This review article shows an analysis of the existing bibliography on resilience, virtual learning and COVID-19, in order to structure and compare the main topics that were covered at the time, in this way to be able to propose new research ideas, to be able to give accurate information to the investigative world. Resilience considered as an essential aspect of well-being that plays an important role in education, knowing that several personal and social factors affect resilience, in addition to virtual learning that goes into making teaching tools for the benefit of education, since it is necessary to provide attention to curriculum development and use new educational methods such as virtual education to strengthen information literacy competence in university students and what education would be like in the face of the crisis of the pandemic caused by COVID-19. That is why this study investigated the effect of resilience in students for virtual learning in times of 
pandemic. Finally, he proposes some alternative solutions for future research, such as the practice of resilience to be able to relate to good virtual learning in this situation of a pandemic, which as a result of this crisis becomes very important the attitude that every student puts to take advantage of and achieve online learning, in this way it is suggested that you should take advantage of the good attitude that the student puts in order to get ahead in any eventuality.

Keywords: Resilience, virtual learning, COVID-19.

\section{INTRODUCCIÓN}

La buena actitud y la capacidad de toda persona para superar circunstancias traumáticas como la que estamos viviendo a causa de la COVID-19, está siendo la resiliencia una novedosa perspectiva sobre el desarrollo humano controlando ciertos impulsos negativos, para tomar como una oportunidad para crecer como persona. Por otro lado, la COVID-19 llegó para cambiar el escenario donde se respira aun incertidumbre y desolación, pero también heroísmo cotidiano. Son muchos los sectores que poco a poco en total respeto de las recomendaciones de aislamiento social, comienzan a brindar soluciones y a sumar desde su lugar. Así se da uno de los sectores más importantes como es la educación, en ese sentido se dice que la formación no se detiene que el aprendizaje; es impostergable es por ello que se han implementado estrategias de educación virtual en pocos días. De esta manera, el estudiante pasa a ser el protagonista indiscutible, es así que en la educación virtual el estudiante bajo la orientación del docente va utilizar muchos de los recursos que brindan los entornos digitales para acceder a información valiosa y realizar actividades que le permitan afianzar el conocimiento y desarrollo de competencias. Por otro lado, la pandemia actual resaltó desigualdades y duras realidades que viven los estudiantes alrededor del mundo.

Después de revisar la bibliografía relevante, se considera tomar las ideas principales de cada autor y contrastar cada uno, por lo que el presente artículo en la parte de su desarrollo considerando como puntos: 1.1 la resiliencia; 1.2 aprendizaje virtual y en el punto 1.3 COVID-19. Finalmente, se concluye con un análisis de los patrones sobresalientes de los estudios revisados y con una propuesta para investigaciones futuras.

A causa de la rápida propagación de la COVID-19, la mayor parte del mundo está experimentando cambios que afectan el sistema educativo. En este caso el cierre de las instituciones educativas afectó a más del

$70 \%$ de la población estudiantil mundial, es por ello que las actividades didácticas tuvieron que transferirse dentro de los entornos virtuales, este cambio de lo físico a lo virtual. Según Giovannella (2021), los docentes no se sentían lo suficientemente capacitados para el manejo del Internet, y solo se limitaban al uso del correo electrónico, ellos no estaban completamente preparados para rediseñar y adaptar su didáctica a la nueva situación. Por su parte, los estudiantes consideran que es muy útil el uso de tecnologías didácticas para aumentar el nivel de su competencia digital, y aceptan positivamente el uso de las tecnologías didácticas y la educación a distancia. Para Gandolfi (2021), en la actualidad más de mil millones de estudiantes están fuera de la escuela debido a esta pandemia, donde la mayoría de ellos están usando prácticas de aprendizaje remoto que presentan inconvenientes de difícil control hasta que se cuente con una vacunación masiva que permita frenar las olas de contagio, pero hasta que ello suceda todos los factores involucrados (sociedad, educación, medios de comunicación, comunidad, etc.); deben centrarse en el manejo, educación y prevención de la COVID-19 (Reynosa Navarro et al., 2021). 


\section{DESARROLLO}

\section{Resiliencia}

La resiliencia es entendida como el resultado del equilibrio entre algunos factores de riesgo, factores protectores y la personalidad del individuo. Asimismo, se define como la capacidad que tiene el individuo para adaptarse a situaciones adversas (Cabanyes, 2010; Cachón et al., 2020; Vizoso, 2019). También, la resiliencia tampoco es estática, puede modificarse en relación al tiempo y a los cambios del contexto (Caldera et al., 2016) y está en relación directa con la satisfacción con la vida (Condori \& Martínez, 2019). En ese sentido la resiliencia como factor, está asociada a la calidad de vida del individuo (Hidalgo \& González, 2019). La resiliencia es una causa de los factores que generan la motivación de logro (Falikah et al., 2020). En ese sentido, es de singular importancia para el logro de los aprendizajes de los estudiantes de los diferentes niveles educativos. Por ejemplo, en el nivel escolar, la etapa resiliente, presenta tres casos positivos: ambiente escolar, el entorno familiar y el autoconcepto (Cajigal et al., 2020). Asimismo, se sostiene que los comportamientos resilientes de los estudiantes se relacionan con el afrontamiento exitoso de las adversidades (Vera, 2014). En el plano social, la resiliencia se ha convertido en una de las herramientas más importantes, para que el individuo se adapte a contextos adversos. En esa línea, el apoyo social se relaciona con la resiliencia (Gaxiola \& González, 2019). Por otro lado, la resiliencia se relaciona con el bienestar psicológico (Benavente \& Quevedo,

2018). Por ello, se sostiene que es un constructo para el análisis del éxito académico (Lozano et al., 2020). Asimismo, constituye una ayuda para que los estudiantes sean más tolerantes y puedan comprender con más claridad el contexto (Santiago et al., 2020). La situación actual de pandemia es uno de los acontecimientos más extremos que está enfrentando la sociedad, tanto por su expansión afectando a millones de personas en todo el planeta, como en su duración en meses y su probable continuidad y consecuencia. Al respecto Cleland et al. (2020) sostienen que la situación ha incluido todos los ámbitos de nuestro funcionamiento como sociedad. Es por ello que se tomó la medida del confinamiento de millones de personas y se trasladaron las actividades al formato virtual. De este modo, el espacio virtual pasa a convertirse en el nuevo entorno educativo, donde el estudiante debe poner en funcionamiento todas sus estrategias cognitivas, afectivas y motivacionales para autorregular su aprendizaje del modo más exitoso posible (Polo et al., 2021). Por lo tanto, se aplican varias iniciativas para brindar una educación flexible, abierta y en línea (Huang et al., 2020). En ese orden, las personas confrontan sus fortalezas, frente a diversos desafíos y exigencias del entorno, en el plano académico y psicosocial, poniendo a prueba sus potencialidades y habilidades (De la Fuente et al., 2017).

Aprendizaje virtual

La pandemia también impactó fuertemente en la educación, un sector muy importante a nivel mundial, y de esta manera millones de estudiantes fueron afectados tras el cierre de las escuelas y centros universitarios debido a la emergencia, así todas las autoridades tomaron la decisión de optar por aplicar la cuarentena, para prevenir que el virus se extienda entre los estudiantes. De esta manera, cambió la forma de trabajo para los docentes como para los estudiantes, ya que todo el proceso de enseñanza y aprendizaje se llevaría desde casa, adaptando así ciertas herramientas como es el uso del WhatsApp con fines educativos. De esta manera, se traslada la educación tradicional a una educación virtual, considerando así algunos aspectos: (1) la disponibilidad de una conexión a Internet en todos los hogares de los estudiantes y profesores; (2) la disponibilidad de una computadora para la educación, o (3) un dispositivo móvil con conexión a Internet (Parra \& Granda, 2021). Antes de esta situación de emergencia, los docentes no estaban familiarizados con los sistemas tecnológicos, en este contexto es de suma importancia explorar y usar dichas herramientas para favorecer el proceso de enseñanza y aprendizaje en este nuevo contexto situacional. Asimismo, las paralizaciones y distanciamiento social durante esta crisis han provocado grandes cambios en la vida de cada persona. Se ha fomentado cada vez más tiempo el uso de dispositivos tecnológicos para poder acceder una educación en línea teniendo así un contacto limitado con las personas. Ahora existe la preocupación del uso del Internet por parte de la población infantil y las posibles consecuencias de este uso, pero se debe tomar en 
cuenta como parte integral en la vida de la mayoría de las personas (Quiñones \& Adams, 2020). Los docentes pueden fomentar un clima positivo de competencias y participación entre sus pares. Sin embargo, se necesita más investigación para comprender mejor la tecnología y el papel de los docentes en la implementación del uso de tecnología en el entorno del proceso de enseñanza-aprendizaje. Es por ello que se dice, las tecnologías digitales son una importante herramienta de aprendizaje que permite a los estudiantes crear una situación social colectiva de desarrollo que integra mundos imaginarios, reales y virtuales, dentro de los juegos virtuales. Por otro lado, la rápida migración al aprendizaje en línea debido a la pandemia de COVID-19, ha generado perturbaciones extremas para los estudiantes, los educadores y el personal administrativo. Esta disrupción surgió y se extendió a sus vidas normales fuera de las instituciones académicas y la universidad (Mohmmed et al., 2020). Por lo tanto, es necesario incorporar un enfoque alternativo, para garantizar que los estudiantes reciban un apoyo integral con estos nuevos desafíos.

Pandemia COVID-19

La educación superior, por supuesto, no ha sido una excepción. A nivel global, nacional y local; el impacto es similar a otras actividades humanas y también asume formas particulares por las características específicas de las actividades docentes, de investigación y de extensión de este nivel educativo (Rojas, 2020). Los actores sociales con universidades, colegios e institutos superiores y centros de investigación, han tenido que hacer frente con premura y creatividad al reto de reorganizar sus actividades para dar continuidad al ejercicio de sus funciones sustantivas y para seguir atendiendo los retos y problemas que enfrentaban desde antes de que estallara la crisis del COVID-19 (Ordorika, 2020). En adición -como resultado esperable de un proceso de esta magnitud a nivel mundial, regional, nacional y local- la COVID-19 ha afectado profundamente a las instituciones, actores y procesos que tienen lugar en la educación superior. Como en otros espacios y actividades de la sociedad se han producido afectaciones y cambios cuya duración y trascendencia son difíciles de prever. Parece conveniente, sin embargo, superar una primera etapa de reacciones obligadas, inevitablemente apresuradas y aceleradas, para dar paso a reflexiones cuidadosas sobre el futuro de la educación superior. Es así que estas reflexiones deben compaginar demandas, proyectos e iniciativas de cambio que estaban teniendo lugar antes de que se desatara la actual crisis, con los análisis de las transformaciones sociales, políticas, económicas y culturales que serán resultados de la propia crisis. Este es un camino y una postura deseable para afrontar los retos que afrontará la educación superior en nuestro país.

\section{CONCLUSIONES}

El estudiante es consciente que los valores y actitudes que tuvieron que desarrollar ante el cambio de modalidad en la educación son autonomía, responsabilidad, paciencia, una mejor administración de los tiempos para cumplir con las tareas y obligaciones académicas. En sentido general hubo un reconocimiento explícito del esfuerzo y la dedicación mostrada por el profesorado al hacer frente a la compleja crisis ocasionada por la contingencia. Entre tanto, docentes y estudiantes reflexionan en torno a la importancia de las tecnologías de la información y la comunicación, como medios indispensables para enfrentar la actual emergencia sanitaria y garantizar la continuidad educativa. Se reconoce la necesidad de fortalecer los mecanismos institucionales para apoyar a los actores educativos ante situaciones de este tipo como la pandemia, con espacios y recursos que ayuden a la constante capacitación y actualización en temas vinculados al uso y manejo de tecnología con fines educativos y ambientes virtuales de aprendizaje. Asimismo, asegurar el derecho a la educación de todas las personas en un marco de igualdad de oportunidades es la primera prioridad y, por consiguiente, todas las decisiones políticas que afecten, directa o indirectamente al sector de la educación superior deberían estar presididas por este derecho. Y, finalmente, prepararse con tiempo para la reanudación de las clases presenciales, evitando la precipitación y ofreciendo los procesos y los mecanismos diseñados para reemprender las actividades docentes. 


\section{Referencias}

Benavente, M. H., \& Quevedo, M. P. (2018). Resiliencia, bienestar psicológico y afrontamiento en universitarios atendiendo a variables de personalidad y enfermedad. Revista de Psicología y Educación Journal of Psychology and Education, 13(2), 99-112.https://doi.org/10.23923/rpye2018.01.161

Cabanyes, J. (2010). Resilience: An approach to the concept. Revista de Psiquiatría y Salud Mental, 3(4), 145151. https://doi.org/10.1016/j.rpsm.2010.09.003

Cachón, J., López, I., San Pedro, M. B., Zagalaz, M. L., \& González, C. (2020). $\square$ e importance of the phoenix bird technique (resilience) in teacher training: CD-risc scale validation. Sustainability, 12(3), 113. https:// doi.org/10.3390/su12031002

Cajigal, E., Hernández-Marín, G. J., Yon, S. E., \& Arias, L. (2020). Resilience of tutors. a case of the faculty of educational sciences of the Universidad Autónoma del Carmen, México. Formación Universitaria, 13(2), 3952. https://doi.org/10.4067/S0718-50062020000200039

Caldera, J. F., Aceves, B. I., \& Reynoso, O. U. (2016). Resilience in university students. A comparative study among different careers. Psicogente, 19(36), 227-239. https://doi.org/10.17081/psico.19.36.1294

Cleland, J., McKimm, J., Fuller, R., Taylor, D., Janczukowicz, J., \& Gibbs, T. (2020). Adapting to the impact of COVID-19: Sharing stories, sharing practice. Medical Teacher, 42(7), 772-775. https:// doi.org/10.1080/0142159X.2020.1757635

Condori, K. O. V., \& Martínez, R. A. (2019). Resilience as a factor determining satisfaction with life among university students. Revista Cubana de Educación Médica Superior, 33(3), 1-16. https://www.medigraphic.com/cgi-bin/ new/resumen.cgi?IDARTICULO=93206

De La Fuente, J., López-García, M., Mariano-Vera, M., Martínez-Vicente, J. M., \& Zapata, L. (2017). Personal self- regulation, learning approaches, resilience and test anxiety in psychology students. Estudios Sobre Educación,

32, 9-26. https://doi.org/10.15581/004.32.9-26

Falikah, T. Y., Nuryana, Z., Yuliana, A. T. R. D., \& Akhmad, F. (2020). Factors affecting education motivation achievement among social support and resilience. Universal Journal of Educational Research, 8(7), 3109-3118. https://doi.org/10.13189/ujer.2020.080739

Gandolfi, A. (2021). Planning of school teaching during COVID-19. Physica D: Nonlinear Phenomena, 415, 132753. https://doi.org/10.1016/j.physd.2020.132753

Gaxiola, J. C., \& González, S. (2019). Perceived support, resilience, goals, and self-regulated learning in high school students. Revista Electrónica de Investigación Educativa, 21(1), 1-10. https://doi.org/10.24320/ REDIE.2019.21.E08.1983

Giovannella, C. (2021). Effect induced by the COVID-19 pandemic on students perception about technologies and distance learning. Smart Innovation, Systems and Technologies, 197, 105-116. https:// doi.org/10.1007/978-981-15-7383-5_9

Hidalgo, C. A., \& González, F. (2019). $\square$ e treatment of acquiescence and the factorial structure of the brief resilience scale (BRS) in Mexican and Chilean university students. Anales de Psicología, 35(1), 26-32. https:// doi.org/10.6018/analesps.35.1.297781

Huang, R., Tlili, A., Chang, T. W., Zhang, X., Nascimbeni, F., \& Burgos, D. (2020). Disrupted classes, undisrupted learning during COVID-19 outbreak in China: application of open educational practices and resources. Smart Learning Environments, 7(1), 1-15. https://doi.org/10.1186/s40561-020-00125-8

Lozano, A., Fernández, J. S., Figueredo, V., \& Martínez, A. M. (2020). Impactos del confinamiento por el COVID19 entre universitarios: Satisfacción Vital, Resiliencia y Capital Social Online. RISE, 9(1), 79-104. https:// doi.org/10.17583/rise.2020.5925 
Mohmmed, A. O., Khidhir, B. A., Nazeer, A., \& Vijayan, V. J. (2020). Emergency remote teaching during Coronavirus pandemic: the current trend and future directive at Middle East College Oman. Innovative Infrastructure Solutions, 5(3), 1-11. https://doi.org/10.1007/s41062-020-00326-7

Ordorika, I. (2020). Pandemia y educación superior. Revista de la educación superior, 49(194), 1-8. http:// resu.anuies.mx/ojs/index.php/resu/article/view/1120

Parra, O., \& Granda, M. F. (2021). Evaluating the Meeting Solutions Used for Virtual Classes in Higher Education during the COVID-19 Pandemic. DOI: 10.5220/0010258201900197 Recuperado de: https:// www.scitepress.org/Papers/2021/102582/102582.pdf

Polo, F. A. E., Pérez, A. R., Aguilar, N. L., \& Reynosa Navarro, E. (2021). Affective pedagogy to stimulate meaningful learning in times of health emergency. RISTI - Revista Iberica de Sistemas e Tecnologias de Informacao, 2021(E40), 166-179. https://www.proquest.com/docview/2483958856?pq- origsite=gscholar\&fromopenview=true

Quiñones, G., \& Adams, M. (2021). Children's Virtual Worlds and Friendships during the COVID-19 Pandemic: Visual Technologies as a Panacea for Social Isolation. Video Journal of Education and Pedagogy, 5(1), 118. Recuperado de: https://pesquisa.bvsalud.org/global-literature-on-novel-coronavirus-2019ncov/resource/en/ covidwho-1090743

Reynosa Navarro, E., Guerra-Ayala, M. J., Casimiro-Urcos, W. H., Vélez-Jiménez, D., Casimiro-Urcos, N. C., Salazar- Montoya, E. O., Casimiro-Urcos, J. F., \& Torres, J. C. C. (2021). Relevance of the mass media in prevention, education and contextual management of COVID-19. World Journal on Educational Technology: Current Issues, 13(1), 129-146. https://doi.org/10.18844/wjet.v13i1.5423

Rojas, L. V., Huamán, C. J. V., \& Salazar, F. M. (2020). Pandemia COVID-19: repercusiones en la educación universitaria. Odontología sanmarquina, 23(2), 203-205. Recuperado de: https:// revistasinvestigacion.unmsm.edu.pe/index.php/odont/article/view/17766

Santiago, M. C., Vergel, M., \& Gallardo, H. D. J. (2020). Resiliencia en estudiantes exitosos en matemáticas. Praxis \& Saber, 11(26), e9973. https://doi.org/10.19053/22160159.v11.n26.2020.9973

Vera, S. (2014). Las Alumnas Universitarias: Modelo De Resiliencia. International Journal of Developmental and Educational Psychology. Revista INFAD de Psicología., 1(6), 143-150. https://doi.org/10.17060/ ijodaep.2014.n1.v6.727

Vizoso, C. M. (2019). Resiliencia, optimismo y afrontamiento en estudiantes de Ciencias de la Educación. Psychology, Society, \& Education, 11(3), 367-377. https://doi.org/10.25115/psye.v11i3.2280 